\title{
Ethics of clinical research and practice in India during the Covid-19 pandemic
}

\author{
SAHAJ RATHI, SP KALANTRI
}

Keywords: COVID-19, pandemic, drugs, research; evidence-based medicine; ethics

\section{Introduction}

Covid-19 has been one of the worst public health calamities faced by humankind in over a century. As of July 23, 2020, there have been 15,633,159 confirmed cases and 635,422 deaths reported, worldwide (1). We are six months into the pandemic, and yet we know little about the disease. The role of medicines is far from optimal, and vaccines are still under trials. Therefore, we have little to defend ourselves against this novel virus.

Drafting health policy around this virus has been a mammoth task. Different parts of India have been in a state of intermittent lockdown since March 2020, yet cases continue to rise. Our highest scientific bodies have approved many drugs for prophylaxis and treatment, based on unconvincing evidence. As an example, hydroxychloroquine (HCQ) is recommended for both prophylaxis and management of Covid-19 in India (2), despite multiple trials (3-7) contradicting any evidence of its efficacy for either indication. Similarly, various experimental therapies (favipiravir, tocilizumab, itolizumab, convalescent plasma) have been approved for clinical use despite an absence of appropriate safety and efficacy data. These approvals are at odds with even the World Health Organization recommendations for approval of emergency use drugs during pandemics (8).

In these desperate times, is it fair to allow the use of new or repurposed drugs without adequate clinical trials? On the other hand, is it practical and ethical to conduct clinical trials before "potentially" helpful treatments can be provided to patients? What is the ethical stand on prescribing experimental therapies to patients? These are difficult questions in any scenario, more so when a country with limited resources is struggling against this pandemic. Moreover, the political and media hype about these drugs seems to be swaying public perceptions much beyond the actual evidence of efficacy, thereby creating a difficult milieu for meaningful research. Here, we attempt to explore the conduct of pandemic research and its clinical implications in the current Indian scenario.

\section{Setting the scene: Expectations from medicine in the Covid era}

The novel coronavirus is primarily a respiratory pathogen, typically resulting in mild symptoms. In less than $20 \%$ of cases, it can cause disease severe enough to cause breathing difficulty, leading to multisystem involvement and death in 3-5\% of cases. Most of those who develop a severe disease are elderly, or have comorbid illnesses. Mortality rates are lower than $0.5 \%$ in known cases among those under 50 years of age (9).

Optimising public health policy has been the crux of this pandemic. The virus is novel, and there are no proven therapies. There is a great need to generate reliable data on both epidemiology and therapeutics. Published research, mostly from Europe, North America and the Asia-Pacific Region shows considerable variability in the epidemiology of infection and severity in different populations (10). Despite the similar time of emergence of the virus, the peak of the epidemic was seen much sooner in Europe than the South East Asian region. The case fatality rates have also varied considerably, being up to 12-14\% in Belgium and the UK, 3-5\% in India and the USA, and less than 1\% in many Middle-Eastern countries. It is not clear what accounts for this geographic variability - the host, the virus, or the environment. Therefore, it is crucial to keep track of international data regularly, as well as generate local data to inform public health decisions. With vastly different socioeconomic structures, age-distributions,

Authors: Sahaj Rathi (sahajrathi@gmail.com), Visiting Consultant, Department of Medicine, Mahatma Gandhi Institute of Medical Sciences, Sevagram, Maharashtra, 442102 INDIA; SP Kalantri (spkalantri@mgims.ac.in), Director-Professor of Medicine and Medical Superintendent, Mahatma Gandhi Institute of Medical Sciences, Sevagram, Maharashtra, 442102 INDIA.

To cite Rathi S, Kalantri SP. Ethics of clinical research and practice in India during the COVID-19 pandemic. Indian J Med Ethics. 2020 Jul-Sep; 5(3) NS: 175-80 DOI: 10.20529/IJME.2020.080.

CIndian Journal of Medical Ethics 2020 
comorbidity profiles, and non-Covid illness demographics between countries, a one-size-fits-all approach would be suboptimal at best, and downright dangerous at worst.

In terms of therapeutics, we have had little to go on. While there are precedents from pre-clinical evidence and older trials of antiviral drugs for related viruses, it would be naïve to presume efficacy without conducting adequately powered randomised controlled trials. These trials need to be placebo-controlled (or against a standard of care, only if clearly established) and assessed specifically on clinically meaningful outcomes. Even if the drugs had been tested for other diseases in the past, they need to be tested from scratch again as they are being repurposed for entirely new indications. Any treatment which does not meet the above criteria may still be offered to patients only after explicit communication of its experimental nature, in a strictly regulated manner.

The disease has a finite duration, ending in recovery or death by the end of hospitalisation in most cases. So far, long-term complications are not known. Therefore, the only outcomes that should matter for potential therapeutics should be in-hospital survival for severe cases, and progression to severe disease in mild cases. Also, length of stay in the intensive care unit or hospital is a valid secondary endpoint in terms of resource utilisation. Duration of milder symptoms, subjective feelings of improvement, viral clearance, or radiological clearance of shadows are not valid endpoints to judge therapeutic efficacy in this disease. Therefore, new or repurposed drugs or interventions need to be judged on clinically meaningful robust, quantifiable endpoints.

Finally, it all comes down to clinical decisions on the ground by treating physicians. It is nearly impossible for busy practitioners to keep up with the tsunami of rapidly evolving evidence of variable quality, which is currently being generated. It is up to the regulatory bodies to distil the evidence and establish clear recommendations, allowing only proven interventions for general use. Interventions with unclear evidence should be strictly restricted to clinical trials until proven effective. Such regulations would enable simpler, heuristic decision-making algorithms, and greatly reduce the expenditure towards ineffective yet fancy therapies. While this approach may seem restrictive to physician freedom, it is possibly the only way to provide a standard of care in a widely unregulated healthcare infrastructure as we have in India. It would also help balance an inequitable resource consumption for Covid-19, which has likely led to an unmitigated crisis of healthcare shortage for all non-Covid illnesses.

\section{Drug regulations in India: Evidence vs recommendations}

In the midst of this pandemic, several drugs have been approved for clinical use by the regulatory bodies, albeit with scarce evidence. If physicians fail to critically appraise the evidence for the safety and benefits of these drugs, potentially harmful therapies might become established as a standard of care. The consequences of such an approach could be dire.

\section{Hydroxychloroquine}

Hydroxychloroquine and chloroquine have been the most debated drugs during the pandemic. Some preclinical and early clinical data suggested possible antiviral action against this coronavirus strain. It is interesting to note that several times in the last three decades, these drugs have been hailed as a potential cure for HIV, dengue, Ebola, Marburg, SARS, Whipple's disease, Q-fever, etc (11). However, the drug has failed to translate into clinical practice for any of these indications (12). As early as March 2020, the Indian Council of Medical Research (ICMR) approved and recommended it for clinical use for pre-exposure prophylaxis (10). This was done even before data from randomised controlled clinical trials were available. The Indian regulatory bodies chose to forgo clinical trials based on in-vitro data and uncontrolled clinical studies available at that time, while nearly all other international drug regulators had restricted access to the drug to trials and special permissions. Instead, a case-controlled study was designed by the scientific body in India to assess the efficacy of prophylaxis (13). This study-underpowered for its end-points and affected by critical biases and confounders - failed to show that HCQ prophylaxis prevented Covid-19 among healthcare workers. Another retrospective study from India also failed to show any effect in pre-exposure prophylaxis of HCQ (14). In the meantime, clinical trials and observational studies from across the world have refuted any beneficial role for this drug in treatment or prophylaxis (3-7). With no benefit and a small but potential risk of serious toxicity, it is unclear why HCQ is still officially recommended for both indications in India (15)

\section{Steroids}

The RECOVERY trial in the UK unequivocally established the mortality benefit of dexamethasone in patients requiring supplemental oxygen at presentation (16). However, there was a trend towards harm when given to those without hypoxia. This is the first intervention found to have a clear mortality benefit and has been able to establish itself as a standard of care in this subset of patients.

In the Indian context, there are two major issues, which need to be addressed. First, in the absence of enforceable prescription regulations, there is a tremendous potential for the misuse of steroids. Abuse of steroids has been a common malpractice among alternative medicine prescribers and quacks for a long time. The media reports of its role in COVID-19 will only add fuel to an already existing fire. Second, the epidemiology of infections in India is very different from the UK. Many experts believe 
Indians have a lower threshold of developing infections on high dose steroids, though there is hardly any published evidence in this regard. Nonetheless, it would be worthwhile to continue observational trials to ensure these results are replicated in our population as well.

\section{Remdesivir}

Remdesivir is a broad-spectrum antiviral drug, found to have some effect in patients with moderate to severe disease. Among the two published trials $(17,18)$, one $(17)$ was able to show moderate clinical benefit-shortening the time to recovery in adults hospitalised with Covid-19. A trend towards lower mortality was also observed, but the difference did not reach statistical significance. These results gave actionable evidence to pursue the use of this drug in clinical practice, albeit with marginal benefits and at a high cost. We need more data to establish or refute its role. Unfortunately, there is not a single trial yet registered for this drug on the CTRI website, but the drug is currently being used, without restrictions, in clinical practice. The perception of its effectiveness has been beyond that suggested by trial data, and there have even been reports of illegal trade (19).

\section{Favipiravir}

Favipiravir is an oral antiviral drug developed in Japan for the treatment of influenza (20). In June 2020, the Drugs Controller General of India (DCGI) provided an accelerated approval for its "restricted emergency use in India" for the treatment of Covid-19 (20). Glenmark, the company marketing the drug in India, claimed that the approval was granted based on the "evaluation of data and expert opinion". However, there was no robust trial evidence to support this stand. An observational study of 80 patients with mild Covid-19, with no control group, published in a journal Engineering, concluded that there was faster disappearance of the virus and quicker improvement of shadows in their chest $\mathrm{x}$-rays (21). There was no data on mortality or progression to severe disease. Additionally, another observational study on favipiravir from Japan (which has not been peer-reviewed) shows that 74\% patients with mild, $67 \%$ with moderate, and $40 \%$ patients with severe disease improved. Favipiravir use resulted in elevated levels of uric acid in $15 \%$ and abnormal liver function tests in $7 \%$ of patients (22). Given that over $80 \%$ of mildly ill Covid-19 patients improve by supportive therapy, the study urged caution in overestimating the benefits of favipiravir for Covid-19 patients.

On July 22, 2020, the results of a small phase 3 clinical trial of 150 patients from India with mild to moderate disease were released. The primary outcome was time to viral clearance, which is meaningless in a clinical context. Nevertheless, the study failed to show any significant advantage of the drug for this outcome (23). Yet, despite the small sample size, and failure to show a significant reduction in time to viral clearance, the results have been marketed as a "success." The results are still only available as a short press release (23). Favipiravir is now in routine clinical use all over the country. This drug costs 12,500 for a 14-day course, with no evidence of meaningful benefit. Again, the regulatory bodies have made no efforts to inform the public about the experimental nature and the potential adverse events of this drug.

\section{Monoclonal antibodies: Itolizumab and Tocilizumab}

Tocilizumab and Itolizumab are both monoclonal antibodies, which block the inflammatory cascade, potentially preventing multi-organ dysfunction. Neither of these drugs has any published randomised controlled trial to endorse their role in Covid-19. While there is some retrospective evidence on Tocilizumab, no such evidence is yet available for Itolizumab.

Recently Biocon Ltd conducted an open-label phase 2 study to test Itolizumab $(n=20)$ with the usual care $(n=10)$. The data on this study is not yet in the public domain; however, according to Biocon, none of the 20 patients assigned to Itolizumab died; 3 of the 10 patients assigned to usual care died. While encouraging, such a low sample size has a high risk of a chance association. At best, this is proof of concept for a proper safety and efficacy study. However, the DCGI granted an emergency use authorisation for Itolizumab, waived the phase 3 trial, and asked Biocon to generate and report data on adverse events from the phase 4 study (24). This decision is baffling, for close to a third of the drugs found promising in phase 2 studies fail in phase 3 studies. Phase 2 data are not definitive enough to determine if a drug works. Phase 4, post-marketing surveillance, is meant to find out the adverse effects of the drug that may become evident after the drug enters clinical practice, and doctors begin to use it extensively. Rarely are phase 4 surveys published, and there is a good chance we may never know the true benefits and risks of this drug now that it has been approved for clinical practice. Huge media publicity has made it harder to test these drugs in randomized controlled trials because no patient would like to be assigned to the control arm.

Covid-19 patients are at high risk of developing secondary bacterial infections, and treatment with Itolizumab may increase this risk. Tocilizumab, another interleukin 6-blocker was shown to result in serious infections-mostly ventilator-associated pneumonias -in half the Covid patients who received the drug (25). This risk is not negligible. Itolizumab, also an interleukin 6-blocker, is aggressively being promoted in India with scant regard for its cost-effectivity and risk-benefit ratio. It is important to ask how patients would fare when the drug is administered in ICUs that have suboptimal infection control measures, settings in which potent antibiotics are either unavailable or unaffordable, and there are limited human resources to care for such patients whose hospital stay is prolonged by hospital-acquired infections. We couldn't agree more with Benjamin Rome and Jerry Avorn who put it succinctly, "For the COVID-19 pandemic, the health of individual patients... will be best served by remaining true to 
our time-tested approach to clinical trial evidence and drug evaluation, rather than cutting corners and resorting to appealing yet risky quick fixes." (26)

\section{Alternative medicines}

There are hundreds of unregulated supplements and formulations being sold under the garb of "immunity boosters". There is no way to verify such claims, as there is no yardstick of immunity in modern medicine. Whether they work on innate or adaptive immunity, humoral or cell-mediated, are hardly a concern for those making such claims. Alternative medicines and health supplements are poorly regulated, and often prey upon the naivety of consumers by making extravagant claims through media. Unfortunately, the absence of effective modern medicine provides the perfect milieu for snake-oil salesmen to abuse the situation. Some have gone too far, claiming to have completed human clinical trials even before animal trials, even though such a trial would be violating numerous ethical standards. The media is rife with products claiming to cure Covid without any proof, and such "herbal" supplements are flying off the shelves $(27,28)$.

\section{Vaccine for Covid-19: Accelerated approval vs cautious approach}

The only true end of this epidemic can come by way of achieving herd immunity either by natural infection or by effective vaccination. All other measures only reduce the number of infections at a certain time point. Overall numbers, in the long run, are likely to be the same. As Covid-19 spreads rapidly, it is imperative to develop a vaccine as early as possible. Many pharmaceutical companies have already come up with their versions of vaccines, and are executing rapid clinical trials. Typically, vaccines require years of research and testing before reaching the public, but during this pandemic, scientists have shown that they could collapse six years of work into six months (29). This has indeed been a remarkable feat. However, vaccines now need thorough safety and efficacy trials before being disseminated to the masses.

It is up to the regulatory bodies to ensure that the vaccines establish beyond doubt that they work, and are safe. However, setting up of unrealistic deadlines by the administration may weaken some of the safeguards, and simply enable the pharma industry to shorten their trials. This of course means higher profits, so they would happily comply. However, vaccines are meant for the otherwise healthy, and flouting of basic safety and efficacy norms may have disastrous consequences. Any major adverse events discovered thereafter will be heavily magnified in the public eye, and may erode public faith in medicine. This may have dire repercussions on other vaccination programmes as well. Therefore, it is absolutely crucial to ensure that accelerated approvals do not come at the cost of overall patient safety.

\section{Healthcare and administrative myopia}

Since the beginning of this pandemic, Covid has been the prime focus of doctors, journalists, healthcare activists, and policymakers alike. India has been struggling with an inadequate healthcare infrastructure since long before this disaster struck. Non-availability of admission beds, especially in intensive care units, has been a usual occurrence in most government hospitals. Due to the lack of proper data, the impact of Covid-19 on all the other diseases is yet unclear. Exclusive allocation of intensive care beds to Covid cases may have grossly compromised care of topical fevers, snakebites, insecticide poisonings etc, which often affect the young, and have a high recovery rate. Tuberculosis and early cancers, which are highly treatable, may have been ignored far too long as they were not immediately life threatening. Unfortunately, as all eyes are focussed on Covid-19, there is no way of knowing how many lives are being lost in this trade-off. Moreover, the economic consequences of stringent lockdowns would have repercussions on public health, nutrition, vaccination policies, and healthcare infrastructure for years to come.

It seems almost sacrilegious to question the policies put in place to limit the spread of the disease. However, the impact of these policies on non-Covid illnesses has to be quantified to ensure equity of healthcare. A predictive model suggests that if the current pattern of spread holds true, India would have around 9 million cases by March 2021 (30). Considering a case fatality rate of 3\%, this would amount to 270,000 deaths over a year of this pandemic. While this is a substantial number, it would still account for less than $3 \%$ of all deaths in India annually (31). Tuberculosis, malaria, and diarrhoea alone account for over $10 \%$ of annual deaths in India when all communicable disease programmes are operational (32). A slight worsening in deaths due to these diseases would be enough to offset any benefit of the herculean efforts against Covid-19. Granted such extrapolations have their limitations; however, they raise some meaningful questions about a possibly myopic view against non-Covid illnesses. The principle of equitable justice in healthcare cannot be ignored. Lastly, while administrative policies have been very active in sensitising the public about social distancing and enforcing it, there has been a dearth of information about the various drugs and therapies. Patients perceive any intervention advised by the doctors as essential, and this belief is reinforced by repeated media propaganda about the "efficacy" of still-experimental antivirals, monoclonal antibodies, and convalescent plasma. Unfortunately, "informed consent" in our country is not always as informed as it should be. As a result, patients or their caregivers may often unconsciously be coerced into treatments of unclear efficacy, violating the ethical principles of patient autonomy. 
It would be reasonable to take a step back and reorient ourselves at this stage of the crisis. This would involve strict reforms in drug approvals based on tangible evidence, ongoing surveillance of efficacy and safety of approved drugs, careful cost-benefit analyses for each intervention, and ensuring rational resource allocation to mitigate preventable morbidity and mortality among non-Covid illnesses.

\section{Conclusion}

This Covid-19 pandemic has disrupted lives globally, and we are still far from having an effective cure or vaccine. The panic and confusion around this epidemic may have led to the lowering of standards of medical research. A flawed trial or misplaced recommendation in these times could potentially translate rapidly into practice, and harm thousands of people before it is caught and rectified. Furthermore, diversion of public health resources towards treatments with questionable benefits will cause preventable harm to those with non-Covid illnesses as well, especially in resource-constrained countries.

The lack of effective therapeutic options should not be an excuse to offer "something" of uncertain efficacy. It erodes the trust of the patient, as it may involve a less-than-honest portrayal of the role of intervention. Above all, the lack of clear benefit heavily skews the risk-benefit ratio, violating the principle Primum non-nocere - the dictum at the heart of all medicine.

Desperate times created by an ongoing pandemic do not justify relaxation of scientific rigor. Au-contraire, they call for the highest and strictest scientific and ethical standards.

\section{References}

1. Worldometers.info. Date unknown [cited 2020 Jul 23]. Available from: https://www.worldometers.info/coronavirus/ Accessed on 23 July 2020.

2. Ministry of Health and Family Welfare, Govt of India. Clinical Management Protocol: COVID-19.Version 5. 2020 Jul 3 [cited 2020 Jul 21$]$. Available from: https://www.mohfw.gov.in/pdf/UpdatedClinicalManagementProtocolforCOVID19dated03072020.pdf.

3. Boulware DR, Pullen MF, Bangdiwala AS, Pastick KA, Lofgren SM, Okafor EC, et al. A randomized trial of hydroxychloroquine as postexposure prophylaxis for COVID-19. N Engl J Med. 2020 Jun 3 [cited 2020 Jul 23]. Epub ahead of print.

4. Cavalcanti AB, Zampieri FG, Rosa RG, Azevedo LCP, Veiga VC, Avezum A, et al. Hydroxychloroquine with or without Azithromycin in Mild-to-Moderate Covid-19. NEng/ J Med. 2020 Jul 23 [cited 2020 Jul 23]. Epub ahead of print.

5. Chen Z, Hu J, Zhang Z, Jiang S, Han S, Yan D, et al. Efficacy of hydroxychloroquine in patients with COVID-19: results of a randomized clinical trial.MedRxiv. 2020:2020.03.22.20040758.

6. Horby P, Mafham M, Linsell L, Bell JL, Staplin N, Emberson JR, et al. Effect of hydroxychloroquine in hospitalized patients with COVID: Preliminary results from a multi-centre, randomized, controlled trial. Preprint. 2020 Jul 15 [cited 2020 Jul 19]. Available from: https://www.medrxiv.org/content/10.1101/20 20.07.15.20151852v1

7. Skipper CP, Pastick KA, Engen NW, Bangdiwala AS, Abassi M, Lofgren SM, et al. Hydroxychloroquine in nonhospitalized adults with early COVID-19. Ann Intern Med. 2020 Jul 16 [cited 2020 Jul 23]. Epub ahead of print.

8. World Health Organization. Off label use of medicines for COVID-19:Scientific brief.2020 Mar 31[cited 2020 Jul 14]. Available from:https://www.who.int/ news-room/commentaries/detail/off-label-use-of-medicines-for-COVID-19.

9. Wu Z, McGoogan JM. Characteristics of and important lessons from the Coronavirus disease 2019 (COVID-19) outbreak in China: Summary of a report of 72314 cases from the Chinese Center for Disease Control and Prevention. JAMA Intern Med. 2020 Feb 24. [Epub ahead of print].

10. Our World in Data. Corona Virus Pandemic Data Explorer. 2020 [cited 2020 Jul 25]. Available from: https://ourworldindata.org/coronavirus-dataexplorer

11. Rolain J-M, Colson P, Raoult D. Recycling of chloroquine and its hydroxyl analogue to face bacterial, fungal and viral infections in the 21 st century. Int $J$ Antimicrob Agents. 2007; 30(4):297-308.

12. Tricou V, Minh NN, Van TP, Lee SJ, Farrar J, Wills B, et al. A randomized controlled trial of chloroquine for the treatment of dengue in Vietnamese adults. PLoS Neg/Trop Dis. 2010;4(8):e785.

13. Chatterjee $P$, Anand T, Singh K, Rasaily R, Singh R, Das S, et al. Healthcare workers and SARS-CoV-2 infection in India: A case-control investigation in the time of COVIDCOVID-19. Indian J Med Res. 2020; 151(5):459-67.

14. Jha S, Soni A, Siddiqui S, Batra N, Goel N, Dey S, et al. Prevalence of flu-like symptoms and COVID-19 in healthcare workers from India.J Assoc Physicians India. 2020;68(7):27-9.

15. National Taskforce for COVID-19. Advisory on the use of hydroxy-chloroquine as prophylaxis for SARS-CoV-2 infection. 2020 Mar 22 [cited 2020 Jul 19 ]. Available from: https://www.mohfw.gov.in/pdf/AdvisoryontheuseofHydroxychloroquinasprophylaxisforSARSCoV2infection.pdf.

16. RECOVERY Collaborative Group, Horby P, Lim WS, Emberson JR, Mafham M, Bell JL, et al. Dexamethasone in Hospitalized Patients with COVID-19 Preliminary Report. N Eng/ J Med. 2020 Jul 17 [cited 2020 Jul 23]. doi: 10.1056/NEJMoa2021436. Epub ahead of print.

17. Beigel JH, Tomashek KM, Dodd LE, Mehta AK, Zingman BS, Kalil AC, et al. Remdesivir for the treatment of COVID-19- Preliminary report. NEng/ J Med. 2020 May 22;NEJMoa2007764. doi:10.1056/NEJMoa2007764. Epub ahead of print.

18. Wang Y, Zhang D, Du G, Du R, Zhao J, Jin Y, et al. Remdesivir in adults with severe COVID-19: a randomised, double-blind, placebo-controlled, multicentre trial.Lancet. 2020;395:1569-78. doi: 10.1016/S0140-6736(20)31022-9

19. Zargar AR. Shortage of key coronavirus drug remdesivir fuels illegal market in India. CBS News. 2020 Jul 22 [cited 2020 Jul 22]. Available from: https:// www.cbsnews.com/news/shortage-of-key-coronavirus-drug-remdesivir-fuels-black-market-in-india.

20. Glenmark gets DCGI nod for favipiravir use in Covid-19. Hindu Business Line. 2020 June 19 [cited 2020 Jul 25]. Available from: https://www. thehindubusinessline.com/companies/glenmark-gets-dcgi-nod-for-favipiravir-use-in-covid-19/article31871629.ece.

21. Cai Q, Yang M, Liu D, Chen J, Shu D, Xia J, et al. Experimental treatment with favipiravir for COVIDCOVID-19: An open-label control study. Engineering (Beijing). 2020 Mar 18[cited 2020 Jul 18]. Available from: https://doi.org/10.1016/j.eng.2020.03.007

22. Favipiravir Observation Study Group. Preliminary Report of the Favipiravir Observational Study in Japan. Aichi, Japan; Fujita Health University; 2020 May 15[cited $2020 \mathrm{Jul}$ 24]. Available from: http://www.kansensho.or.jp/uploads/files/topics/2019ncov/covid19_casereport_en_200529.pdf

23. Glenmark Pharmaceuticals Ltd. Press Release. Glenmark announces top-line results from phase 3 clinical trial of favipiravir in patients with mild to moderate COVID-19 2020 Jul 22[cited 2020 Jul 23]. Available from: https://www.glenmarkpharma.com/sites/default/files/Glenmark-Announces-TopLine-Results-From-Phase-3\%20-Clinical.pdf. 
24. PTI.Biocon gets DCGI nod for use of Itolizumab for treatment of COVID-19 patients. Economic Times. 2020 Jul 11 [cited 2020 Jul 23]. Available from:https:// health.economictimes.indiatimes.com/news/pharma/biocon-gets-dcgi-nod-for-use-of-itolizumab-for-treatment-of-covid-19-patients/76916677

25. Somers EC, Eschenauer GA, Troost JP, Golob JL, Gandhi TN, Wang L, et al. Tocilizumab for treatment of mechanically ventilated patients with COVID-19. Clin Infect Dis. 2020 Jul 11; ciaa954. doi: 10.1093/cid/ciaa954. Epub ahead of print.

26. Rome BN, Avorn J. Drug Evaluation during the COVID-19 Pandemic. N Engl J Med. 2020;382(24):2282-4. doi: 10.1056/NEJMp2009457.

27. Karindalam A. Increase in intake of Ayurvedic immunity boosters as COVID-19 cases rise. The Week. $2020 \mathrm{Jul} 11$ [cited 2020 Jul 22]. Available from: https:// www.theweek.in/news/health/2020/07/12/increase-in-intake-of-ayurvedic-immunity-boosters-as-COVID-19-cases-rise.html.

28. Rao SR. Immunity-boosting Vitamin C tablets in short supply, say Bengaluru chemists. Times of India. 2020 Jul 16[cited 2020 Jul 22]. Available from: https://imesofindia.indiatimes.com/city/bengaluru/immunity-boosting-vitamin-c-tablets-in-short-supply-say-chemists/articleshow/76987623.cms.

29. Jackson LA, Anderson EJ, Rouphael NG, Roberts PC, Makhene M, Coler RN, et al. An mRNA Vaccine against SARS-CoV-2 — Preliminary Report.NEngl J Med. 2020 Jul 14. Epub ahead of print

30. Computational Mathematics Group (CMG). PDE-based modelling of COVID-19 infections. Bangalore, India; CMG, Indian Institute of Science. Updated 2020 Jun 18 [cited 2020 Jul 22]. Available from: https://cmg.cds.iisc.ac.in/COVID/

31. World Bank. Death rate, crude (per 1,000 people) - India. 1960-2018 [cited 2020 Jul 22]. Available from: https://data.worldbank.org/indicator/SP.DYN. CDRT.IN?locations=IN.

32. Ministry of Home Affairs. Government of India. Office of the Registrar General \& Census Commissioner. Causes of Death Statistics. New Delhi; MoHA;2020. Available from:https://censusindia.gov.in/vital_statistics/causesofdeath.html. Accessed on July 22, 2020.

\section{DNAR Guidelines: Supporting end-of-life decisions}

\section{OLINDA TIMMS}

\section{Keywords: DNAR, end of life decisions, surrogate decisions, patient autonomy}

The Indian Council of Medical Research Consensus Guidelines on 'Do Not Attempt Resuscitation' (DNAR) were published in the April 2020 issue of the Indian Journal of Medical Research (1), and simultaneously in the National Medical Journal of India (2). It is a timely effort at resolving a long-standing clinical dilemma.

DNAR orders are not uncommon in hospital situations at end of life, and while they are legally permissible in many countries, this remains a grey area in India. This policy document attempts to formalise the modalities of a difficult clinical decision that has hitherto been an informal part of professional practice in this country. The guidelines describe the conditions in which DNAR orders may apply, and provides a flow chart of decision-making. It also includes a patient information sheet, FAQs, and a DNAR consent form.

Physicians were always acutely aware of the futility of resuscitation measures in some end-of-life situations, and generally followed the process outlined in this guideline, but it was done informally as there was no medico-legal sanction for the decision. Clinicians are convinced of the necessity of DNAR orders for a sub-group of terminally ill patients, but are wary of social or peer repercussions in this ethical minefield. With these guidelines, doctors will proceed with more confidence, even if the legal liability is not specifically addressed in the guidelines.

To that end, the ICMR tag lends authority to the document, but it still begs the question of why guidelines on clinical practice are being formulated by the National Research Council. The Medical Council of India (Board of Governors) or National Medical Commission that currently regulates medical education and practice should ideally have addressed this aspect of clinical care, as addendum to its ethics regulations. The paucity of practising clinicians and critical care specialists on the "expert group" (1) that created this policy is surprising, and the reasons for this will remain a matter of speculation. Such a document that impacts professional decision-making in clinical end-of-life settings would surely have benefitted from clinical expertise from different parts of the country, through regional consultations. Also conspicuous by their absence are patient groups; directly affected by these guidelines, whose voices and opinions need to be heard. Unfortunately it is top down approaches such as these that have led to confusion and distrust in healthcare, even litigation, when all viewpoints are not represented.

Not surprisingly, some practical details are left unaddressed in the document. There is reference to the "treating physician" but it is unclear who this will be in many hospitals where patients are admitted under "Units" and all doctors of the Unit may see the

Author:Olinda Timms (olindatimms@gmail.com), Division of Health and Humanities, St Johns Research Institute, Bengaluru 560034 INDIA.

To cite:Timms O. DNAR Guidelines: Supporting end-of-life decisions. Indian J Med Ethics. 2020 Jul-Sep; 5(3) NS: 180-1. DOI:10.20529/JJME.2020.081.

OIndian Journal of Medical Ethics 2020 